\title{
Intra-nasal glial heterotropia in an infant: The first case report from Himalayan region of India
}

\author{
Manu Malhotra $^{1 *}$, Priyanka Goirala ${ }^{2}$, Saurabh Varshney ${ }^{3}$, Shalini Rao $^{4}$, Rashmi Malhotra $^{5}$ \\ ${ }^{1,5}$ Additional Professor, ${ }^{2}$ Senior Resident, ${ }^{3,4}$ Professor, ${ }^{1-3}$ Dept. of Otorhinolaryngology, ${ }^{4}$ Dept. of Pathology, ${ }^{5}$ Dept. of Anatomy, All India \\ Institute of Medical Sciences, Rishikesh, Uttarakhand, India
}

*Corresponding Author: Manu Malhotra

Email: manumalhotrallrm@gmail.com

\begin{abstract}
Introduction: Nasal glial heterotropia is a rare developmental anomaly in which normal mature glial tissue presents as intranasal and/or extranasal masses. It can present in all ages, though it is more commonly diagnosed in paediatric age groups. Only a limited number of cases are reported worldwide.

Aim: To report a rare case of intra- nasal glial heterotropia in an infant.

Case Description: The authors here report the first case from Himalayan region in India. The condition presented in an infant and was diagnosed with the help of clinical, ultraonographic Doppler, Computerised Tomographic Scans and histopathology. The case was treated with complete surgical excision.

Conclusion: Nasal glial heterotropia, though rare, should be considered in the differential diagnosis of a nasal mass in a young paediatric patient. The diagnosis can be reached with clinical, radiological (Sonography Scans /CT/MRI) and histopathological methods, including immunohistochemistry. Finally, complete surgical excision is the treatment of choice.
\end{abstract}

Keywords: Nasal glial heterotropia, Nasal glioma, Encephalocele, Nasal mass, Glial fibrillary acidic protein.

\section{Introduction}

Nasal glial heterotopia (NGH) refers to congenital malformations of displaced normal, mature glial tissue, which are no longer in continuity with an intracranial component and present as intranasal or extra-nasal masses. These are rare congenital lesions which occur sporadically with no familial tendency or sexual predilection and are frequently diagnosed in newborn infants, however, they may rarely be found in adults also. NGH are different from encephaloceles, which are a herniation of brain tissue and/or leptomeninges, that develops through a defect in the skull, where there is a continuity with the cranial cavity. ${ }^{1-3}$

Histologically, NGH are composed of astrocytes and neuroglial cells interlaced with fibrovascular connective tissue that is covered by the epithelium or respiratory mucosa. It may be arranged in a lobular pattern, and cystic structures may be present as well. The picture may vary in places, and may be difficult to identify with hematoxylin-eosin stain alone; special stains and immunohistochemistry are thus of great utility when making the diagnosis. ${ }^{4,5}$

Though the reported incidence is 1 in every 20,000 to 40,000 births, a little more than approximately 250 cases have been reported so far. ${ }^{3,6}$ Few cases have been reported from India. However, there has been no case report from Himalayan region of India. We here report a case of NGH in an infant which presented with a nasal mass since birth and was treated surgically.

\section{Case Presentation}

An eleven month old child presented with a nasal mass present since birth, associated with history of prominent mouth breathing and recurrent nasal discharge. There was no history suggestive of epistaxis and meningeal irritation. The swelling was firm, non-tender, non-pulsatile, noncompressible and non-reducible with pressure. The swelling progressively increased to present size of $1.5 \mathrm{~cm}$ (Fig. 1). This mass showed no growth or change in size during crying or jugular vein compression (Furstenberg sign). Hypertelorism was absent. There was no other abnormality detected in ear, nose, throat and physical examination. All routine haematological and biochemical parameters were within normal range.

Ultrasonographic Doppler study revealed a non-vascular mass. A computed tomography (CT) scan of the facial bones showed an iso-dense shadow, which occupied the left nasal cavity with slight blockage of osteo-meatal complex. There was no communication with frontal lobe of brain, bony defect in Crista Galli or erosion of the bony walls of nose (Fig. 2). Fine needle aspiration was attempted twice which yielded scanty hemorrhagic aspirate only. Since no conclusion could be drawn from the cytology, excision of the swelling was performed under general anaesthesia and the nasal cavity was examined with nasal endoscope post-operatively for any remnants (Fig. 3). 
On gross examination the mass was greyish, well circumscribed of $1.5 \mathrm{~cm} \mathrm{X} 1 \mathrm{~cm} \mathrm{X} 1 \mathrm{~cm}$ in dimensions. Cut section was solid homogenous and grey-white. No areas of hemorrhages, cyst or calcification was seen. Microscopy revealed fibrocollagenous tissue, muscle fibers with intervening areas of glial tissue having few astrocytes with fibrillary cytoplasm. (Fig. 4) Special staining with Van Gieson's, highlighted the collagen around glial tissue. Histopathologist suggested the diagnosis of NGH, though immunohistochemistry for Glial fibrillary acidic protein (GFAP) was also performed to confirm the diagnosis.

The post-operative period was uneventful and patient was discharged in satisfactory condition on fifth day. The patient was symptom free when examined after 4 months of fallow up.

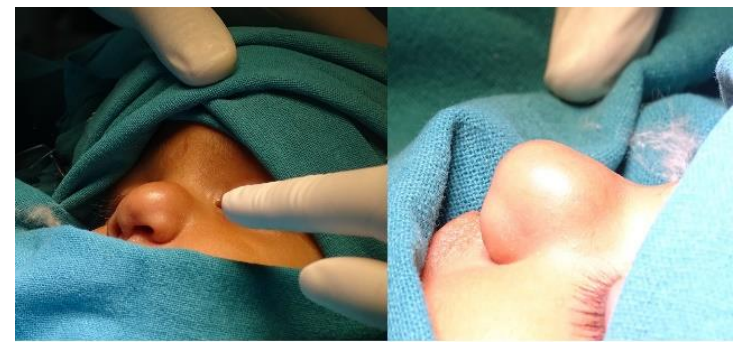

Fig. 1

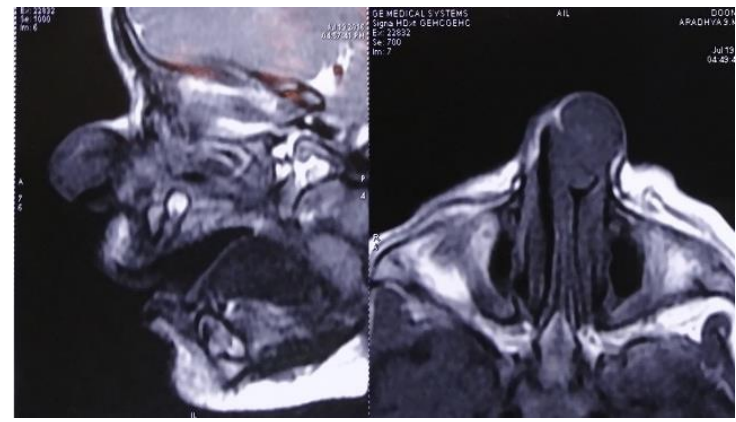

Fig. 2

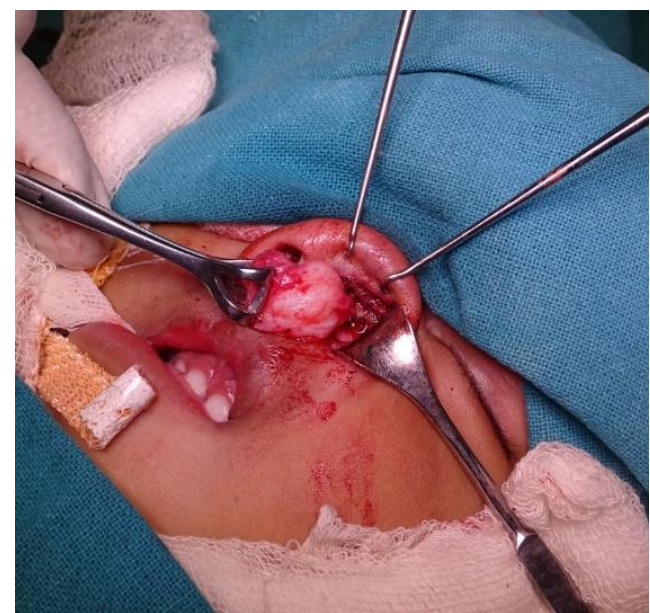

Fig. 3
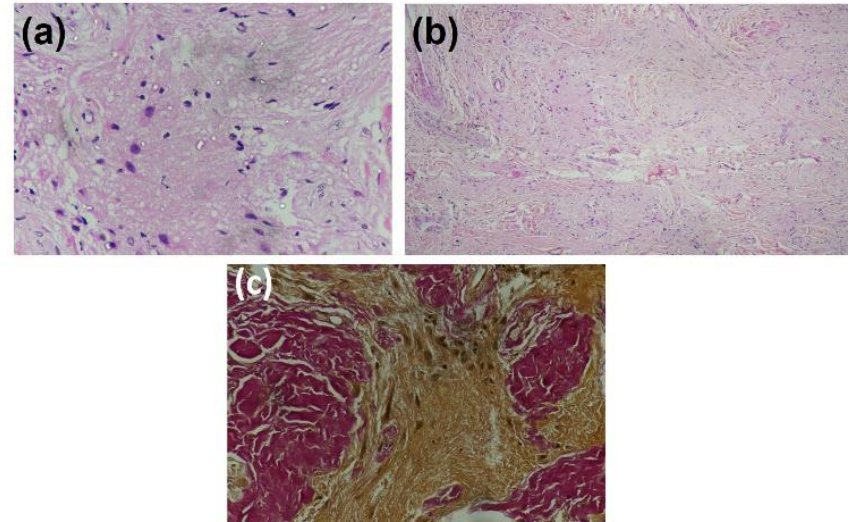

Fig. 4

\section{Discussion}

NGH, or differentiated neural tissue outside the cranial vault is very uncommon. Heterotopic brain tissue has also less commonly been reported to occur in other sites, such as the pharynx, lung, orbits, palate, tongue, cheek, lip, and neck. Nasal encephaloceles and NGH appear to share a similar embryogenic origin. They occur when the neuroectodermal and ecto-dermal tissues fail to separate during the development of the nose. NGH might link the intracranial dura mater through the foramen cecum and evidence of this connection is observed in about $10-15 \%$ of histopathological studies and referred as a "fibrous stalk". ${ }^{6}$

NGH has varied clinical presentations. The extranasal form (60\%) is superficially located, and expands into the subcutaneous space. Intranasal NGH are less common, accounting for $30 \%$ of all cases, usually seen at birth and often present with respiratory distress in the early life. Intranasal form may involve the cribriform plate also. Mixed form $(10 \%)$ associates both extranasal and intranasal extensions. $^{6}$

The differentiation of NGH from encephalocele is based on the presence or absence of the connection between the mass and the intracranial tissue. High resolution computed tomography (CT) and/or magnetic resonance imaging (MRI), are helpful in this discrimination. CT is useful for identifying small bone defects at the skull base, whereas MR imaging, with its multiplanar capabilities, is excellent for identifying communication with the adjacent brain. Furthermore, in light of the developmental abnormality, bony defects may also be seen in association with nasal gliomas while still showing no communication with the brain parenchyma. The distinction may be made by the presence of meningitis and/or cerebrospinal fluid rhinorrhoea either before or after surgical manipulation. Incisional or aspiration biopsies are not recommended without establishing connection between brain and tumour with the help of scans, as they carry the risk of neurologic complications, including meningitis or damage to 
functional brain tissue. The other differential diagnoses are vascular tumors, mainly hemangiomas, which are the most common vascular tumors in infancy. Ultrasound can be useful for determining whether the mass is cystic or solid, and Doppler flow studies can provide further information regarding the arterial flow patterns. Unlike hemangiomas, which show high arterial Doppler flow velocity during the end-diastolic phase, NGH demonstrate low arterial flow velocity during the end-diastolic phase. ${ }^{1,3,6}$

Definitive diagnosis is established by histopathologic evaluation of the lesion which shows fibrillary neurological tissue with a prominent network of glial fibers, gemistocytic type astrocytes predominantly in background of neuropil, representing classic neuroglial tissue with varying degree of glial fibrosis. The histology of nasal glial heterotopia may be difficult to identify with hematoxylin and eosin stain alone. Special stains and immunohistochemistry, such as glial fibrillary acid protein (GFAP) immunostain, which enhances the glial tissue within the background fibrosis, and positive synaptophysin, which confirms the presence of neuronal cells, are thus of great utility when making the diagnosis. ${ }^{2-5}$

Surgical excision of the tumour is the treatment of choice. However, inadequate excision may result in recurrence in $4-10 \%$ of the cases. Endoscopy may be useful in avoiding tumour remnants.

\section{Conclusion}

$\mathrm{NGH}$, though rare should be considered in the differential diagnosis of a nasal mass in a young paediatric patient. The diagnosis can be reached with clinical, radiological (Sonography Scans /CT/MRI) and histopathological methods, including immunohistochemistry. Finally, complete surgical excision is the treatment of choice.

\section{Declaration on conflict of interest and ethical standards}

Authors declare that they have no conflict of interest. This article does not contain any studies with human participants or animals performed by any of the authors. All other applicable international, national, and/or institutional ethical guidelines have been followed.

\section{References}

1. Penner CR, Thompson L. Nasal glial heterotopia: A clinicopathologic and immunophenotypic analysis of 10 cases with a review of the literature. Ann Diagnostic Pathol. 2003;7(6):354-9.

2. Hagiwara A, Nagai N, Ogawa Y, Suzuki M. A case of nasal glial heterotopia in an adult. Case Rep Otolaryngol. 2014;2014:354672. doi:10.1155/2014/354672.

3. Kardon DE. Nasal glial heterotopia. Arch Pathol Lab Med. 2000;124(12):1849.

4. Cerda-Nicolas M, Sanchez Fernandez de Sevilla C, LopezGines $\mathrm{C}$, et al. Nasal glioma or nasal glial heterotopia? Morphological, immunohistochemical and ultrastructural study of two cases. Clin Neuropathol. 2002;21:66-71.

5. Khade MG, Maloker DD, Patil RN. Nasal Glial Heterotropias: Diagnostic role of histopathology. Sch J Appl Med Sci. 2015;32(F):962-5.

6. Surda P, Hobson J. A rare case of nasal glial heterotopia presenting as sphenochoanal polyp. Int J Otolaryngol Head Neck Surg. 2015;4:77-80.

How to cite this article: Malhotra M, Goirala P, Varshney S, Rao S, Malhotra R. Intra-nasal glial heterotropia in an infant: The first case report from Himalayan region of India. IP Indian J Anat Surg Head Neck Brain. 2020;6(1):27-9. 